\title{
Microprocessor Control of Dc Motor Drives
}

\author{
N.R. Chitragar ${ }^{1}$, S.G. Kulkarni ${ }^{2}$ \\ Assistant Professor, Faculty of Electrical \& Electronics Engineering, Gogte Institute of Technology, Belagavi ${ }^{1}$ \\ Associate Professor, Faculty of Electrical \& Electronics Engineering, Gogte Institute of Technology, Belagavi ${ }^{2}$
}

\begin{abstract}
There are various applications in industry which utilize DC drives to achieve high production levels and high quality standards. One of the common methods for bidirectional motoring control is accomplished by using a twoquadrant switching circuit. Some DC drive applications require braking which can be accomplished economically by using regenerative braking which is achieved by using a four-quadrant switching circuit. This braking scheme is more efficient, economical, and requires less maintenance as compared t o other types of braking. In this paper, the theory of regenerative braking is used to develop a control system for DC drives which is based on the operation of a fourquadrant switching circuit. This microprocessor based system is designed with a new integrated circuit (LMD 18200) which contains a four-quadrant switching circuit and on-board control logic. This system has many industrial and commercial applications and can be modified to conform to specific voltage, current, and horsepower ratings. Applications of this system range from larger industrial drives to small DC drives and servo motors such as those used in robotics, printers, and plotters. The system microprocessor can also be interfaced to other control units for process control applications.
\end{abstract}

Keywords: DC drives, Microprocessor Control, LMD 18200, Servo Motors, Control Systems.

\section{INTRODUCTION}

Recent developments in semiconductor technology have made smaller, faster microprocessors available at reduced costs. This has led to their wide spread use in AC and DC adjustable-speed drive industry [1]. Furthermore, the regulating system of the motors which are used in industrial applications are also required to be more compact so that they are easy to maintain, less expensive, and have extended control features [2].The potential applications for microprocessors $t$ o control some or all of electronic functions justify the use of a microprocessor controller [3]. The microprocessor can also provide monitoring and diagnostic functions that are too costly t $o$ provide with hard-wired circuits [4].

In this paper, a microprocessor based control system for a DC motor, which uses standard hardware, is described. There is a problem when the direction of rotation of large DC motor, operating at high speed, is suddenly changed. The supply current to the motor cannot be switched quickly due to the counter EMF and energy stored in the control circuit. The motor should be slowed down by using some sort of braking technique. This can be done by physically putting a braking device on the shaft of the motor. However, this scheme is not efficient and may be insufficient if the motor is driving a large load. The system discussed in this paper uses a more efficient technique that uses regenerative braking which utilizes the motor as a generator. This is accomplished by using a four-quadrant switching circuit [5]. The motor will force current back to the supply which will reduce the speed of the motor. The system program contains a variable braking delay to ensure the slowing of the motor $\mathrm{t}$ o a speed level that will not produce high surge currents during reversal.

The proposed system features bidirectional motoring, braking, speed control, torque regulation, over-current shutdown, over-voltage shutdown, and over-heating shutdown.

\section{CONTROL SYSTEM}

\subsection{System Features}

The present system is designed for small DC motor applications. Fig. 1 shows the block diagram of the system which has the following features:

8088 microprocessor control [10]

forward and reverse motoring

forward and reverse braking

fiber optic isolation speed control

torque regulation

over-current, over-voltage, and

over-heating shutdown

speed and error code displays

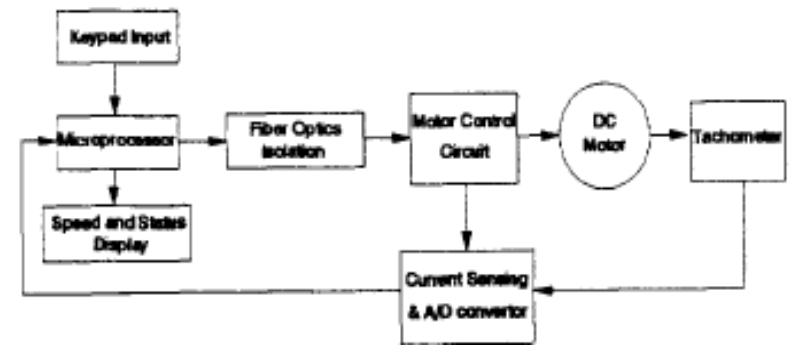

Fig. 1 Control system block diagram

The proposed system is rated at $3 \mathrm{~A}$ and $55 \mathrm{~V}$ DC. These are the limitations imposed by the LMD18200 in the motor control circuit [7]. The system can be expanded to control large DC motors. In addition, it can also be modified to control stepper motors and servo motors for various applications.

\subsection{Four-Quadrant Operation}

The four-quadrant motor control circuit is shown in Fig. 2. This circuit provides four-quadrant control $t$ o a load connected between $\mathrm{t} w$ o pairs of power MOSFETs. The voltage across the motor and the direction of the current through the motor can be of either polarity. 
During forward motoring, the DC motor operates in the first quadrant where the armature voltage and armature current are both positive. During forward regenerative braking, the DC motor operates in the second quadrant where the armature voltage is positive and the armature current is negative. During reverse motoring, the DC motor operates in the third quadrant where the armature voltage and armature current are both negative. During reverse regenerative braking, the DC motor operates in the fourth quadrant where the armature voltage is negative and the armature current is positive $[\mathbf{6}, 9]$.

In regenerative braking, the motor acts like a generator and transfers the kinetic energy of the mechanical load and motor back to the supply. In a separately excited DC motor, the armature and field can be controlled independently to provide the required torque during starting $[6,9]$.

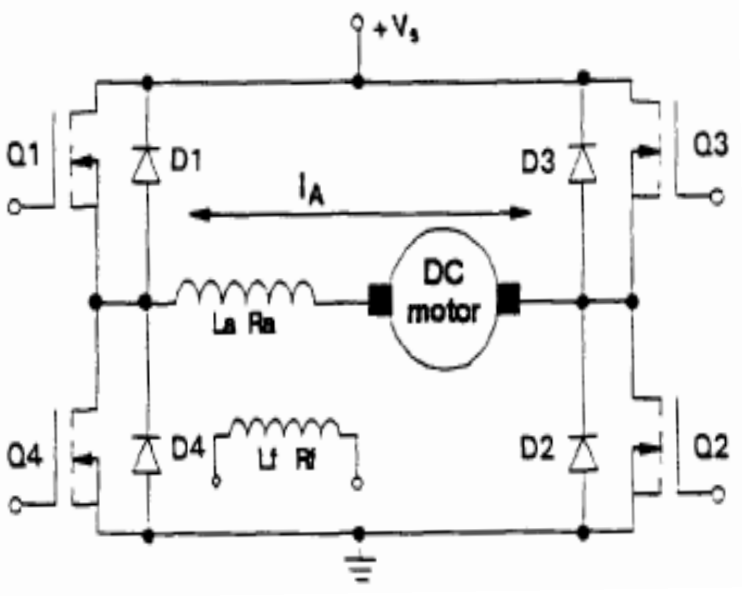

Fig. 2 Four-quadrant DC motor control circuit

Forward Motoring: MOSFETs Q1 and Q2 are turned on while MOSFETs Q3 and Q4 are off. When Q1 and Q2 are turned on together, the supply voltage appears across the motor terminals and the armature current rises. When Q1 is turned off and Q2 is still turned on, the armature current decays through Q2 and D4.

Forward Regenerative Braking: MOSFETs Q1, Q2, and Q3 are turned off when MOSFET Q4 is turned on. The armature current, which rises, flows through Q4 and D2.When Q4 is turned off, the motor acts as a generator and returns energy t o the supply through D1 and D2.

Reverse Motoring: The field current of the motor is reversed while MOSFETs Q3 and Q4 are turned on. MOSFETs Q1 and Q2 are off. When Q3 and Q4 are turned on together, the armature current rises and flows in the reverse direction. When Q3 is turned off and Q4 is still turned on, the armature current falls through 04 and D2.

Reverse Regenerative Braking: The field current is in the same direction as in reverse motoring. MOSFETs Q1, Q3, and Q 4 are off. When Q2 is turned on, the armature current rises through Q2 and D4. When Q2 is turned off, the armature current falls and the motor returns energy to the supply through Q3 and D4 [6].

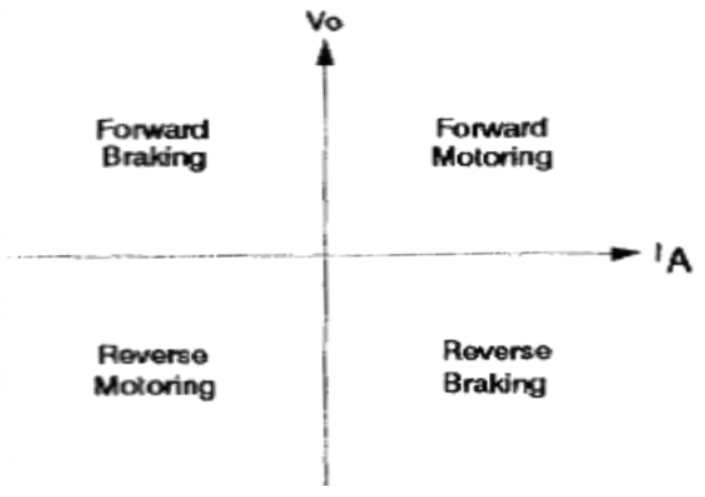

Four-quadrant operation

2.3 Switching Circuit

The motor control circuit is the unique part of the entire system. The circuit houses an integrated circuit (LMD18200) that contains an internal four-quadrant $(\mathrm{H}-$ bridge) circuit. The LMD18200 has on-board logic that controls the switching patterns of the internal power switching transistors. The advantage of using the LMD18200 is its superb accuracy of switching its internal DMOS power devices. In other switching schemes, there is a problem if the gating angles of the switching devices are retarded too far. This situation may cause undesirable current paths which could affect the motor operation. The LMD18200 accepts pulse width modulated (PWM) signals that are used for speed control and torque regulation. There are other capabilities such as current sensing, high voltage sensing and over-heat sensing. It also has a $5 \mathrm{~V}$ control logic which makes it easy to interface to microprocessor systems. There are three inputs which control all motor operations. The basic motor control system is shown in Fig. 3.

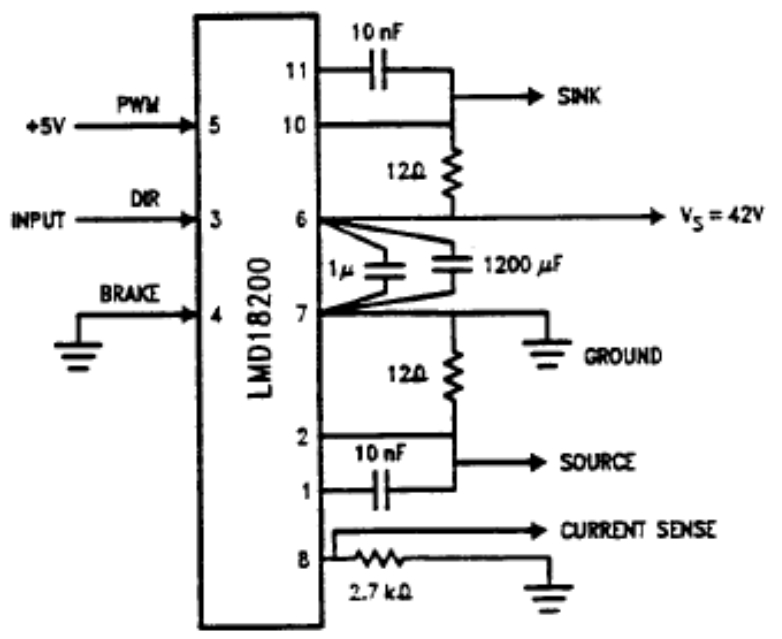

Fig. 3 LMD18200 schematic

The sensing capabilities of the control circuit provide a means of notifying the microprocessor of possible circuitdamaging situations before they occur. Feedback of the sensing $\mathrm{t}$ o the microprocessor is done through the use of AID conversion and by utilizing the microprocessor interrupts. The microprocessor can shut down the system or cut back on the motor control circuit output to correct such situations. 
2.4 Motor Control

The motor control circuit can accept two types of PWM inputs; the first type would utilize a $0 \%$ to $100 \%$ fig. 4 . The control circuit would pass more voltage outputs near $100 \%$, thereby increasing the speed. The output voltage would be minimal near $0 \%$ duty cycle. There are integrated circuits available that produce a PWM output that can be interfaced to the microprocessor to get the required duty cycle needed to achieve the desired motor speed.

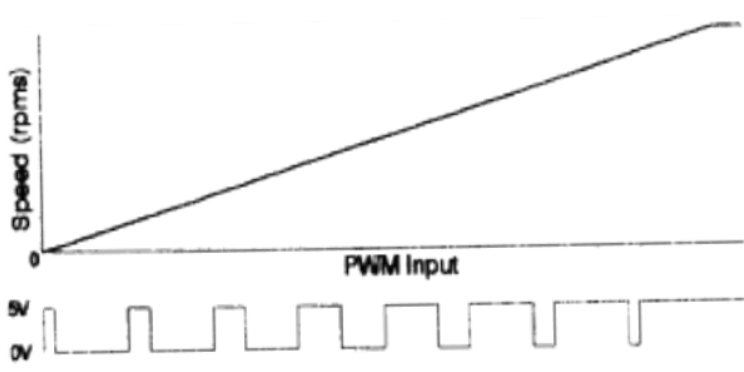

Fig. 4 PWM signal for unidirectional speed control

The second type of signal would be referenced at $50 \%$ duty cycle as shown in Fig. 5. The control circuit would give zero output at $50 \%$ duty cycle. This PWM signal would be driving both the PWM input and direction input of the LMD18200. The control circuit would pass positive output voltage from $51 \%$ to $100 \%$ duty cycle. The higher output would be achieved near the $100 \%$ duty cycle. This could be referenced as speed control for the motor in the forward direction. Inversely, the $0 \%$ t o $49 \%$ ranges would control speed in the reverse direction. The control circuit output would be increasing as $0 \%$ duty cycle was approached. This PWM signal could be produced by using $\mathrm{t} \mathrm{w}$ o programmable timer counters and a flip-flop. One counter would be programmed to pulse at a certain time while the other would pulse according to the microprocessor program. By changing the pulse time of the second counter, the output of the flip-flop will be altered. Complex programming will achieve the variable PWM signal.

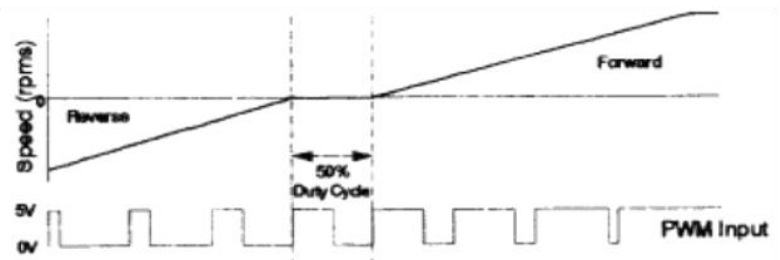

Fig. 5 PWM signal for bidirectional speed control

The advantage of using the second type Of PWM input would be for small DC motors, stepper motors, and servo motors that require very little or no braking at all before changing motoring directions. The first method would be useful for larger motor applications that require braking and more sensitive speed control to prevent high surge currents.

2.5 Surge Current Measurements

One important consideration when designing the system program was the surge current that results while changing motoring directions. The speed of the motor at the time of reversal directly affects the amount of surge current. Not all applications allow the motor to completely stop before starting in the opposite direction. Therefore, depending on the size of the DC motor being driven, surge currents must be considered when designing the system program. The program shown in Fig. 6 is designed with a variable braking delay. This delay was calculated from $42 \mathrm{~V}$ motor test measurements.

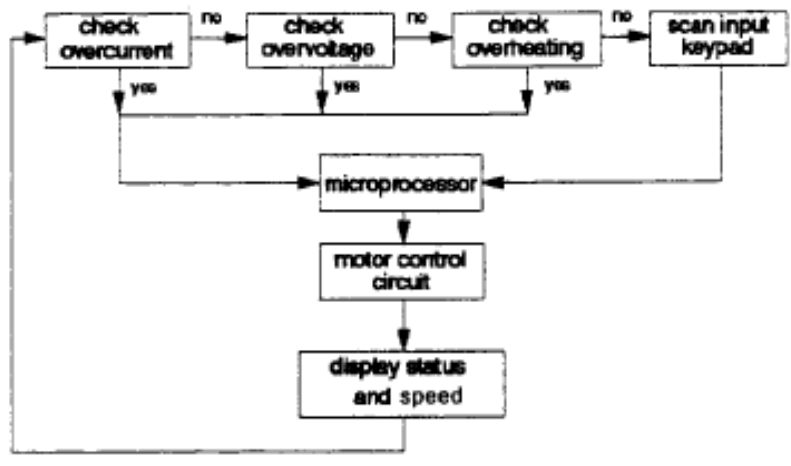

Fig. 6 System control program

The surge current measurements were taken at different speeds while changing motor directions. As a result, the program is designed to slow down the motor to a certain speed before changing directions. The motor control system is capable of handling surge current of $6 \mathrm{~A}$ and a continuous current of $3 \mathrm{~A}$. The test results are shown in fig. 7. The motor control circuit current sensing capabilities are also used to prevent high surge currents. If the surge current or continuous current is too high, the current sensing feedback will tell the microprocessor to adjust the supply voltage and apply braking if necessary.

This variable braking delay is desirable for large dc motor applications. This would allow quick reversal of the motor. Smaller dc motor applications may not require any braking if the developed surge current is relatively small.

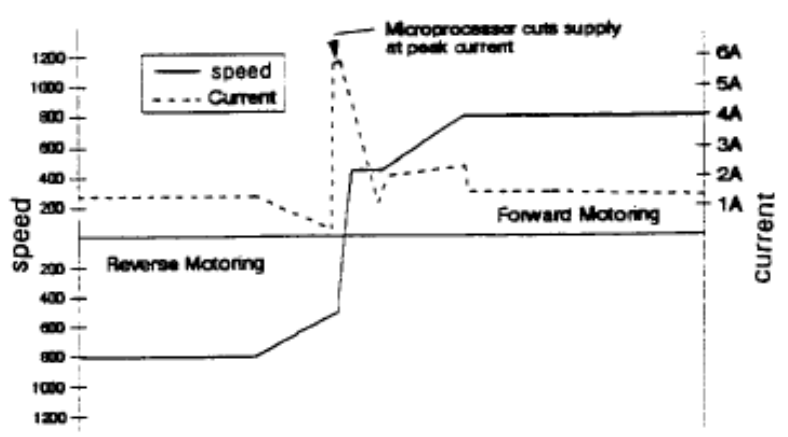

Fig. 7 surge current and speed test results.

\section{SWITCHING CHARACTERISTICS}

The system operating frequency range depends on three different system components as shown in Fig. 8. The system is presently limited by the switching characteristics of the fiber optics circuit. The fiber optics internal oscillator can be upgraded to achieve a wider operating frequency range. The motor control circuit operating frequency can also be improved $500 \mathrm{kHz}$ by connecting 
external 10nF capacitors from the LMD18200 chip outputs to bootstrap pins.

Fast switching capabilities would be a greater requirement for small DC motor applications such as printers. Larger DC motor applications would not require very fast switching characteristics due to the amount of time it inherently takes to accelerate or decelerate a large motor.

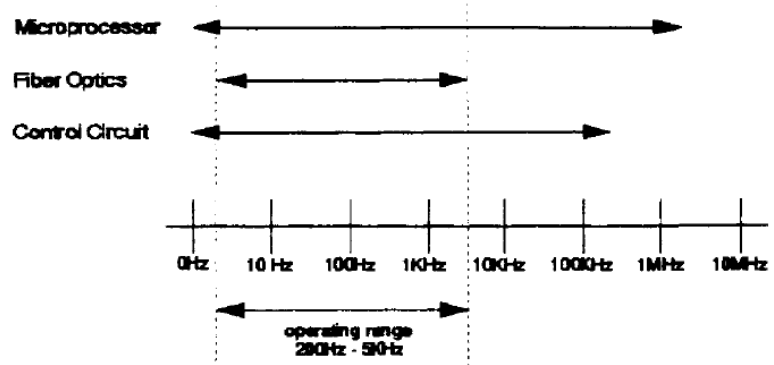

Fig. 8 System operating frequency ranges

\section{DESIGN PARAMETERS}

There are several compatibility considerations to take into account when designing the system. The switching characteristics of the microprocessor, fiber optics and motor control circuit have been discussed in the previous section. The other considerations are the voltage and current sourcing capabilities of each part of the system. The microprocessor system has sufficient fan-out and a 5 $\mathrm{V}$ output which is adequate to drive the fiber optics and display. Further expansion or other applications may require the use of additional hardware on the microprocessor outputs to boost sourcing capabilities. The measured output current of each fiber optics line is 0 to 15 $\mathrm{mA}$. The output voltage of each line was variable $+/-1.0 \mathrm{~V}$. Depending on the supply voltage of the fiber optic system. The LMD18200 requires a minimum of $11.8 \mathrm{~V}$ for its sourced switching voltage before it would begin to pass voltage to its outputs. Maximum output current or voltage could not be tested since the chip has its own internal shutdown when maximum rating levels are exceeded.

\section{SYSTEM CONSIDERATIONS}

One of the unique features of this system is that it can be modified or expanded for use with other motor applications by using additional hardware, feedback loops and program modifications.

5.1 Servo and Stepper Motor Applications

The system can be modified for use with a servo motor or stepper motor. An incremental encoder would be added to the shaft of the servo/stepper motor which will take the place of DC motor. This encoder will provide feedback to the microprocessor which calculates the motor speed, position and direction. The recommended encoder should provide 360 pulses per revolution. This gives an angle of resolution equal to one degree which simplifies system programming.

\subsection{Large DC Motor Applications}

The system can be modified, to control large DC greater motors, by adding three more LMD18200s to the motor control circuit. These LMD18200s would provide output to a network of resistors which would alter the supply voltage and current to drive the gate of high power switching devices such as SCRs. Fig. 9 shows this system modification. The microprocessor would require more i/o ports to monitor feedback from each LMD18200. The fiber optics system would also require additional lines to provide input signals to the LMD18200s. The system program would also be altered to service the additional feedback loops and outputs.

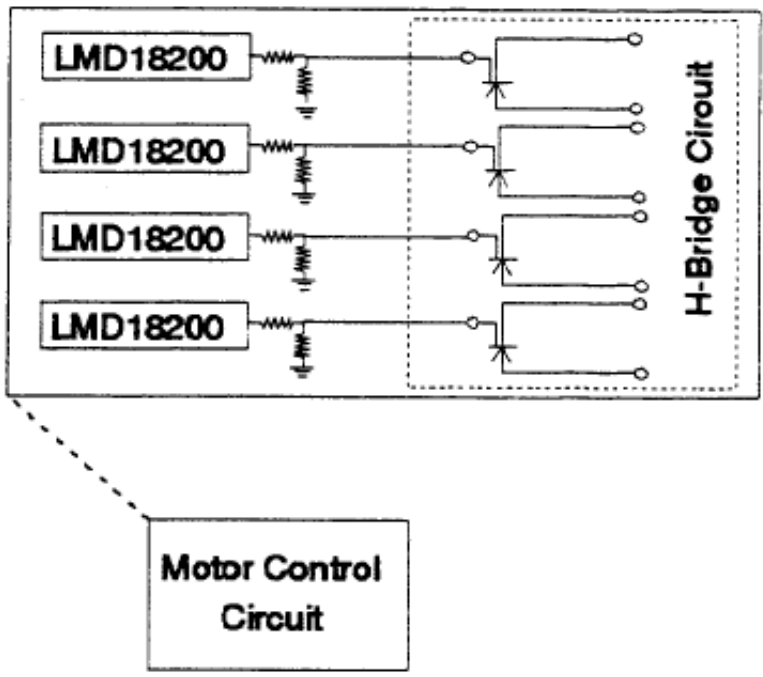

Fig. 9 Modified motor control circuit block diagram

\section{CONCLUSION}

The system discussed in this paper proved to be very efficient for controlling small .DC motor applications. Although the proposed system IS limited to sourcing capabilities of $3 \mathrm{~A}$ and $55 \mathrm{~V}$, it can be modified to be used with many other motor applications. The system also has many other special features, such as its sensing capabilities which can be applied to many control applications. LMD18200 can be added to the control circuit to control any pattern of switching devices. The LMD18200 could also take the place of switching devices in small DC motor applications.

\section{REFERENCES}

[1] I A. Kusko and D. Galler, "Survey of Microprocessor in Industrial Motor Drive Systems," Microcomputer Control of Power Electronics and Drives, pp. 159-162, 1987

[2] T. Konishi, K. Kamiyama and T. Ohmae, "A Performance Analysis of Microprocessor-Based Control System Applied to Adjustable Speed Motor Drives", IEEE industry ADDlicationS, Vol. IA - I 6, NO. 3, pp. 378-387, May 1980.

[3] A. Kusko, "Applications of Microprocessor to AC and DC Electric Motor Drive Systems", IEEE-IAS Conf. Record, Annual Meeting, pp. 1079-1081, Oct. 1977

[4] M. H. Rashid, Power Electronics, Prentice Hall, 1988. 